\title{
Errata: Nonequilibrium Second-Order Phase Transitions in Stochastic Lattice Systems: A Finite-Size Scaling Analysis in Two Dimensions ${ }^{1}$
}

\author{
J. L. Vallés ${ }^{2,3}$ and J. Marro ${ }^{2,4}$ \\ Received December 17, 1987
}

In the paragraph following equation (3.2) on page 97 the expression $x_{x}(T)$ should be corrected to $u_{x}(T)$.

In the last line of page 104 the expression $A_{s}(T) \approx A \varepsilon^{-\sigma}$ should read $A_{\infty}(T) \approx A \varepsilon^{-\sigma}$.

On page $105 x \approx$ const should be changed to $X \approx$ const and Eq. (4.3) should read

$$
X(x) \approx A x^{-\sigma}+A_{s} x^{-\sigma_{s}}
$$

In the caption for Fig. 10 the description of the symbols should be $L=(\triangle)$ 10, (○) 15, (*) 30, (๑) 50, and $(\square) 100$.

Also, in the last paragraph on page 114 the expression for the fit to the longitudinal correlation function should read $g_{l}(r) \approx A \exp (-r / \xi) r^{-b}$.

\footnotetext{
${ }^{1}$ This paper appeared in J. Stat. Phys. $49: 89$ (1987).

${ }^{2}$ Departamento de Física Fonamental, Universitat de Barcelona, E-08028 Barcelona, Spain.

${ }^{3}$ Present address: Courant Institute of Mathematical Sciences, New York University, 251 Mercer Street, New York, N.Y. 10012.

${ }^{4}$ Present address: Departamento de Física Aplicada, Facultad de Ciencias, Universidad de Granada, Spain.
} 